\title{
Reward Sensitivity Predicts Dopaminergic Response in Spatial Neglect
}

\begin{abstract}
It has recently been revealed that spatial neglect can be modulated by motivational factors including anticipated monetary reward. A number of dopaminergic agents have been evaluated as treatments for neglect, but the results have been mixed, with no clear anatomical or cognitive predictors of dopaminergic responsiveness. Given that the effects of incentive motivation are mediated by dopaminergic pathways that are variably damaged in stroke, we tested the hypothesis that the modulatory influences of reward and dopaminergic drugs on neglect are themselves related.
\end{abstract}

We employed a single-dose, double-blind, crossover design to compare the effects of Cocareldopa and placebo on a modified visual cancellation task in patients with neglect secondary to right hemisphere stroke. Whilst confirming that reward improved visual search in this group, we show that dopaminergic stimulation only enhances visual search in the absence of reward. When patients were divided into REWARD-RESPONDERs and REWARD-NON-RESPONDERs, we found an interaction, such that only REWARD-NONRESPONDERs showed a positive response to reward after receiving Co-careldopa, whereas REWARD-RESPONDERs were not influenced by drug. At a neuroanatomical level, responsiveness to incentive motivation was most associated with intact dorsal striatum.

These findings suggest that dopaminergic modulation of neglect follows an 'inverted U' function, is dependent on integrity of the reward system, and can be measured as a behavioural response to anticipated reward. 


\section{Introduction}

Investigators have employed a number of approaches, including drug treatments and noninvasive brain stimulation, in order to improve symptoms in patients with spatial neglect (Cappa, Sterzi, Vallar, \& Bisiach, 1987; Koch et al., 2012; O'Shea et al., 2017; Pizzamiglio et al., 2004; Rossetti et al., 1998). Although some of these treatments, particularly prism adaptation, have shown remarkable results, clinical trials have tended to only demonstrate improvement in a subset of patients (Bowen, Hazelton, Pollock, \& Lincoln, 2013; Pierce \& Buxbaum, 2002; van der Kemp, Dorresteijn, Ten Brink, Nijboer, \& Visser-Meily, 2017). This particularly applies to pharmacological treatments. Noradrenergic and cholinergic agonists have been shown to have positive effects on attention in neglect (Dalmaijer et al., 2018; Lucas, Saj, et al., 2013), but the majority of drug studies have assessed the effects of dopaminergic agents. The dopaminergic system has been of particular interest to investigators because damage to dopaminergic tracts has been shown to cause neglect-like behaviour in animals, and dopamine receptor stimulation has decreased or ameliorated this behaviour (Corwin et al., 1986; Marshall \& Gotthelf, 1979). The majority of clinical reports have demonstrated a broadly positive effect (Fleet, Valenstein, Watson, \& Heilman, 1987; Geminiani, Bottini, \& Sterzi, 1998; Gorgoraptis et al., 2012; Hurford, Stringer, \& Jann, 1998; Mukand et al., 2001), although two studies have shown a worsening of neglect with a single dose of a dopamine agonist (Barrett, Crucian, Schwartz, \& Heilman, 1999; Grujic et al., 1998). Although investigators have examined whether dopamine might boost performance in a specific cognitive domain that is affected in neglect, such as working memory or selective attention, no reliable effects in these aspects of cognition have been found in the most extensive study to-date (Gorgoraptis et al., 2012). It has been suggested that only neglect patients with a specific pattern of neuroanatomical damage would be likely to respond to dopaminergic stimulation e.g., that patients with more anterior damage would be least likely 
to improve. However, neuroanatomical analysis of dopamine responsiveness in neglect has not yet shown any systematic pattern (Gorgoraptis et al., 2012).

Following on from Mesulam's initial anecdotal observation (Mesulam, 1985) of a motivational component to neglect, investigators have recently taken an empirical approach to understanding how motivation, particularly anticipated monetary reward, influences the clinical syndrome of neglect (Lecce et al., 2015; Li et al., 2016; Lucas, Schwartz, et al., 2013; Olgiati, Russell, Soto, \& Malhotra, 2016; Robertson, 2012). Using a modified standard clinical task, we have shown that neglect is improved by anticipated reward in patients without damage to the dorsal striatum (Malhotra, Soto, Li, \& Russell, 2013), a key node in dopaminergic reward-processing circuits (Schultz, 1998). This is in keeping with reports of blunted motivational response in patients with clinical apathy secondary to basal ganglia dysfunction following stroke, and also in Parkinson's Disease (PD) (Rochat et al., 2013; Schmidt et al., 2008; Shore, Rafal, \& Parkinson, 2011). Moreover, in a single case report, Adam and colleagues explored the effect of dopaminergic treatment upon reward-response and apathy secondary to bilateral basal ganglia stroke. They found that both apathy and reward responsiveness improved with dopaminergic stimulation, and that this effect ceased upon treatment withdrawal (Adam et al., 2013), mirroring effects observed in PD (Czernecki et al., 2002; Muhammed et al., 2016).

This evidence suggests an interplay between reward responsiveness and the effects of dopaminergic stimulation, with a possible further link with clinical apathy. Here, we explored whether reward responsiveness and basal ganglia damage play a role in the differential response to dopaminergic stimulation observed in neglect. Specifically, we did this by investigating the effects of dopaminergic stimulation upon response to incentive motivation in a group of patients with left neglect following right hemisphere stroke. In this proof-of-concept study, we employed a randomised, double-blind, single-dose crossover design comparing 
the effects of a single dose of L-Dopa (Co-careldopa) with placebo on the modified standard cancellation task with which we previously demonstrated the effects of anticipated reward on neglect (Malhotra et al., 2013).

Given dopamine's recognised role in reward processing and the variable effects of dopaminergic stimulation in neglect, we hypothesised that response to dopaminergic treatment is linked with sensitivity to incentive motivation in each individual patient, which in turn is dependent on damage to reward-processing circuitry.

In order to address this hypothesis, we aimed to answer the following three questions:

i) Does dopamine induce a reward effect in those individuals with neglect who previously did not manifest such a motivational response?

ii) In those who do exhibit a reward-attention interaction (i.e. improvement of neglect with anticipated monetary reward) without any pharmacological intervention, what effect does subsequent dopaminergic stimulation have on this motivational response?

iii) Are there any anatomical differences between neglect patients with and without a rewardattention interaction? Specifically, does damage to the striatum reliably predict a lack of response to reward?

In addition, given the demonstrated association between reward responsiveness and apathy, we also explored whether individuals with blunted reward-attention responses were more likely to have clinical apathy. 


\section{Methods}

\section{Patients}

Individuals with neglect secondary to first right hemisphere stroke were identified after presenting to the stroke unit at Imperial College Healthcare NHS Trust. Neglect was operationally defined as omitting more than five targets on the Mesulam shape or Behavioural Inattention Test (BIT star cancellation tasks (Mesulam, 1985; Wilson, Cockburn, \& Halligan, 1987), administered on A3 sheets and timed over two minutes (following evidence that healthy individuals under the age of 65 years can complete the shape cancellation task in less than two minutes (Weintraub \& Mesulam, 1988)).

The following exclusion criteria were applied: age $<18$ years; significant sensory and/or motor impairments affecting patients' ability to perform the tests; pre-existing neurological conditions (for example dementia) that would confound cognitive assessments; acute concomitant illness (including cardiac, renal or liver failure); symptomatic postural hypotension; history of psychosis or Parkinson's Disease; antihypertensive medications commenced within the previous two weeks; current exposure to monoamine oxidase inhibitor, dopaminergic or sympathomimetic drugs; stroke within the previous four weeks; pregnancy; breastfeeding.

Eighteen potentially eligible patients with clinical manifestations of neglect were screened and took part in the study, with nine individuals excluded from further analysis after performing at ceiling on the reward cancellation task in the baseline session (see below and Figure 1). Patient demographics are shown in Table 1. All patients provided written informed consent before participating in the study, which was approved by the UK National Research Ethics Service. 


\section{Study Design}

A randomised, double-blind, placebo-controlled study design was employed, consisting of five separate sessions (baseline, 1a, 1b, 2a, 2b) across three days (Figure 1).

In each session, patients performed a cancellation task with separate Reward and No-reward conditions (Malhotra et al., 2013). In the Reward condition, patients had to find and mark 'pound coin' targets amongst featureless distractor stimuli (controlled for hue and luminance) on an A3 sheet of paper, whereas in the No-reward condition target stimuli consisted of brass button targets with appropriate featureless distractors (Figure 2). Before testing, participants were informed that they would receive a monetary reward corresponding to their performance on the Reward condition, and performance on the No-reward condition would not be related to any subsequent reward (The inclusion of the No-reward control allowed for any spontaneous fluctuation in neglect severity). Subjects were asked to circle as many targets as they could find and to inform the examiner when they felt that they had completed the task. A maximum time period of five minutes was allowed per array.

An apathy assessment was undertaken using the apathy evaluation scale (AES) (Marin, Biedrzycki, \& Firinciogullari, 1991). Both the clinician (C) and self-rated (S) versions were administered, and, where possible, the informant (I) version. To determine the motivational characteristics of each individual, the behavioural inhibition/approach system (BIS/BAS) questionnaire of Carver and White (1994) was completed.

Day 1 took place within a week of the baseline session, and was divided into two parts, (a) and (b), with Session $1 \mathrm{~b}$ taking place 60 minutes (the time taken for levodopa to reach peak plasma concentration) after the end of Session 1a. Patients performed both Reward and Noreward conditions of the cancellation task in each of these. 
Either a single oral dose of Co-careldopa 25/100 or placebo was administered to patients at the end of Session 1a. We used Co-careldopa because such a combination of L-Dopa and a peripheral dopa-decarboxylase inhibitor is the most direct method of dopaminergic stimulation (Chong \& Husain, 2016). Furthermore, L-Dopa's lack of specificity for any particular dopamine receptor subsets makes it the ideal candidate to exert dopaminergic effects on neglect, reward response, and apathy, without the potentially more selective effects of a receptor-specific agonist. To counteract the possible side effects of nausea and vomiting, oral Domperidone $20 \mathrm{mg}$ was given at the very beginning of session 1 and 2 .

Day 2 took place within a week of Day 1, but those who received L-Dopa previously were given placebo on this occasion and vice versa. Randomisation was carried out by the pharmacy at Imperial College Healthcare NHS Trust, and both examiners and patients were blind to whether Co-careldopa or placebo was being administered. Within and between individuals, the order in which the Reward and No-reward conditions were performed was counterbalanced between the baseline and other sessions.

To limit practice effects, five different variations (each with equal numbers of targets and distractors, but with jittered spatial positions) of each cancellation array were used, one for each of the five sessions, with the configuration of the Reward and equivalent No-reward conditions being the same in each session. The order in which the five arrays were administered across sessions was counterbalanced across patients.

After completion of both cancellation conditions in each session, subjects were asked to rate their motivational levels for each on a simple visual analogue scale (VAS) composed of a $100 \mathrm{~mm}$ length horizontal black line. They were instructed to place a single vertical mark across the scale at a point which they felt best represented their motivational levels on the No-reward condition in black and on the Reward condition in red, with the left and right 
extremities of the line representing minimum and maximum levels respectively. It should be noted that this scale was being used to test for a difference between subjective motivation levels for the two conditions within individuals, which should not be affected by any rightward bias. Patients received vouchers after completion of both tasks in each session in accordance with their performance, up to a maximum of $£ 10$. As requested by the Ethics committee all patients received an equal sum (total worth £50) by completion of the study.

\section{Analysis}

In our previous study, we found an effect of reward on cancellation task performance in the second session, after participants had received reward, and consistent with the results of experiments in healthy individuals (Anderson, Laurent, \& Yantis, 2011; Bourgeois, Neveu, Bayle, \& Vuilleumier, 2017; Della Libera \& Chelazzi, 2006; Kiss, Driver, \& Eimer, 2009). In order to examine the effects of reward and consequently the effects of L-Dopa on such a reward-attention interaction in this study, those subjects who performed at or above ceiling (defined as finding $>50$ targets) on the reward task in the baseline session were excluded from further analysis. To determine whether or not the effects of L-Dopa differ in the presence or absence of a reward response, we made an a priori decision to divide the remaining subjects into REWARD-RESPONDER and REWARD-NON-RESPONDER groups on the basis of their performance in Session 1a. This subdivision was not affected by the order of presentation of each condition in Session 1a. As in our previous study (Malhotra et al., 2013), the former were defined as those who found more targets in the Reward condition compared to the No-reward condition in Session 1a (and before receiving any medication).

\section{Lesion Anatomy}

Using the MRIcron software package (www.mccauslandcenter.sc.edu/mricro/mricron), each patient's stroke lesion was manually mapped directly by a trained neurologist onto their native clinical CT or MRI (DWI or FLAIR if the images were, respectively, acquired within or 
beyond 48 hours of stroke onset), on all axial slices where lesion was evident. The anatomical scan and lesions were subsequently mapped onto stereotaxic space using Clinical Toolbox for spatial normalisation (www.mccauslandcenter.sc.edu/CRNL/clinicaltoolbox), implemented via the SPM8 software package (www.fil.ion.ucl.ac.uk/spm/software/spm8).

We determined, for each patient, relative lesion volume overlapping regions of interest across the whole brain. These were obtained from reference atlases of Brodmann Areas, cortical and subcortical structures, and white matter tracts (Desikan et al., 2006; Zhang et al., 2010). The proportion of each ROI occupied by lesion was calculated for each subject by matrix multiplication, i.e.: lesion profile (row vector) x ROI (column vector) (Rinne et al., 2013). Such a region-of-interest approach to test anatomical associations respects the fact that cognitive functions are spatially distributed (Gajardo-Vidal et al., 2018). Subject-level values were compared between groups using univariate logistic regression. All analyses were performed in MATLAB (The Mathworks, inc.) with a statistical threshold of $p<0.05$ set for each. 


\section{Results}

There were no adverse events or side effects reported by any of the participants during the study. Repeated-measures ANOVA was the parametric statistical analysis of choice. Input factors were condition (referring to the two levels of No-reward and Reward), drug (referring to the two testing days on which either placebo or L-Dopa was given) and session (referring to the pre-drug (a) and post-drug (b) sessions, regardless of placebo or L-Dopa), with reward response (REWARD-RESPONDERs versus REWARD-NON-RESPONDERs) as a betweensubjects factor. The assumption of homogeneity of variance was tested using Levene's test of equality of variances.

\section{Total Cancellation Performance}

The data for total number of targets found were not normally distributed, as measured using the Kolmogorov-Smirnov test, and were thus reverse-scored followed by a log transformation in order to correct unequal variances. Using the transformed data across all 9 patients, a three-factorial (condition $\mathrm{x}$ drug $\mathrm{x}$ session) repeated measures ANOVA revealed a main effect of condition $\left(\mathrm{F}_{(1,8)}=7.89, \mathrm{p}=0.023, \eta_{p}^{2}=0.50\right)$, with a greater number of targets found in the Reward condition (mean 37.7, SEM 5.3) than in the No-reward condition (mean 35.3, SEM 4.8) (Figure 3A). There was no significant correlation between reward response and baseline cancellation task (Mesulam shape or BIT star task) performance suggesting that there was no relationship between neglect severity and reward responsivity.

There was no main effect of L-Dopa on cancellation performance, but there was a 3-way interaction $\left(\mathrm{F}_{(1,8)}=5.13, \mathrm{p}=0.05, \eta_{p}^{2}=0.39\right)$. Planned contrasts showed that the interaction was due to a condition $x$ drug interaction specifically on post-drug $(b)$ sessions $\left(F_{(1,8)}=7.73\right.$, $\mathrm{p}=0.024), \quad \eta_{p}^{2}=0.49$ ), with significantly more targets found in the No-reward condition following L-Dopa (mean 37.9, SEM 5.6) compared to following placebo (mean 32.7, SEM 
4.5) $\left(t_{(8)}=2.45, p=0.04\right)$, suggesting that $L-D o p a$ modulated neglect in the absence of reward (Figure 3B). By contrast, there was no difference in performance post-placebo versus post-LDopa for the Reward condition $\left(t_{(8)}=0.61, p=0.56\right)$. Therefore L-Dopa did not have a synergistic effect with reward. There were no other main effects or interactions (all $p$ values > $0.08)$.

\section{Cancellation in each Hemispace}

A four-factorial (condition $\mathrm{x}$ drug $\mathrm{x}$ session $\mathrm{x}$ hemispace) repeated measures ANOVA for the number of targets found on each side of the cancellation array revealed a main effect of hemispace, $\left(\mathrm{F}_{(1,8)}=11.42, \mathrm{p}=0.010, \eta_{p}^{2}=0.59\right)$, with fewer targets found on the left (mean 14.5, SEM 3.2) than on the right (mean 21.9, SEM 2.1). However, there was no condition $x$ hemispace interaction $\left(F_{(1,8)}=1.15, p=0.316\right)$. That is, incentive motivation improved performance across the visual space but not specifically in neglected hemispace. Moreover, there was no condition $x$ drug $x$ session $x$ hemispace interaction, $\left(F_{(1,8)}=0.35, p=0.57\right)$. That is, the modulating effects of L-Dopa occurred across visual space and not specifically in neglected hemispace.

\section{REWARD-RESPONDERS versUS REWARD NON-RESPONDERS}

In a mixed-design ANOVA (condition $\mathrm{x}$ drug $\mathrm{x}$ session with reward response as a betweensubjects factor) examining the number of targets found, a condition $\mathrm{x}$ session $\mathrm{x}$ reward response interaction $\left(F_{(1,7)}=7.33, p<0.05\right)$ was present. In post hoc analyses, a condition $x$ reward response interaction in the pre-drug sessions approaching significance was identified $\left(F_{(1,7)}=5.12, p=0.058, \eta_{p}^{2}=0.42\right)$. That is, as would be expected from their performance in Session 1a, in all pre-drug sessions REWARD-RESPONDER patients performed better in the Reward (mean number of targets found 39.6, SEM 6.9) compared to the No-reward (mean number of targets found 35.0, SEM 6.6) condition, whereas REWARD-NON- 
RESPONDERs performed worse in the Reward (mean number of targets found 30.5, SEM 7.8) condition compared to the No-reward (mean number of targets found 35.8, SEM 7.4) condition.

A significant session $x$ reward response interaction for the reward condition $\left(F_{(1,7)}=5.46\right.$, $\left.\mathrm{p}=0.05, \eta_{p}^{2}=0.44\right)$ was also present. During the Reward condition, the performance of REWARD-RESPONDERs was better in the pre-drug sessions (as expected) compared to REWARD-NON-RESPONDERSs but not different in post-drug sessions, with the performance of REWARD-NON-RESPONDERs improving from pre- (mean number of targets found 30.5, SEM 7.8) to post- (mean number of targets found 39.3, SEM 9.0) drug sessions.

There was also a near-significant drug $\mathrm{x}$ session $\mathrm{x}$ reward response three-way interaction $\left(F_{(1,7)}=5.27, p=0.055, \eta_{p}^{2}=0.43\right)$. Post hoc tests performed to determine the source(s) of this interaction suggested that it could be accounted for by a session $\mathrm{x}$ reward response interaction for the L-Dopa treatment day $\left(\mathrm{F}_{(1,7)}=8.91, \mathrm{p}=0.02, \eta_{p}^{2}=0.56\right)$ (Figure $\left.3 \mathrm{C}\right)$. That is REWARD-RESPONDER performance was worse following L-Dopa whereas REWARDNON-RESPONDERs performed better, specifically on the Reward task $\left(t_{(3)}=3.04, p=0.056\right)$.

A further five-factorial (condition $x$ drug $x$ session $x$ hemispace $x$ reward-response) ANOVA for the number of targets found in each hemispace revealed main effects of condition, session and hemispace but no interaction involving hemispace and reward-response.

\section{Subjective Motivation and Apathy}


Using a repeated measures ANOVA, we examined subjective motivation in the pre-placebo combined with pre-L-Dopa conditions only (to exclude any potential drug effects). Condition $x$ reward-response repeated measures ANOVA revealed a main effect of reward $\left(F_{(1,7)}=11.01\right.$, $\mathrm{p}=0.013, \eta_{p}^{2}=0.61$ ), with mean VAS scores for Reward (mean 79.65, SEM 5.76) being higher than mean VAS scores for No-reward (mean 57.63, SEM 7.67). There was no condition $x$ reward-response interaction $\left(\mathrm{F}_{(1,7)}=0.16, \mathrm{p}=0.70, \eta_{p}^{2}=0.023\right)$. There was no significant difference between REWARD-RESPONDERs and REWARD-NON-

RESPONDERs $\left(\mathrm{F}_{(1,7)}=0.86, \mathrm{p}=0.39, \eta_{p}^{2}=0.11\right)$. Non-parametric analysis (data for the reward post-L-Dopa session were not normally distributed) of all VAS scores (See Table 2) using Mann-Whitney $U$ tests showed no differences between the VAS scores of the REWARDRESPONDER and REWARD-NON-RESPONDER groups, for each of Reward and Noreward conditions for each of the pre-placebo, post-placebo, pre-L-Dopa and post-L-Dopa sessions. Both REWARD-RESPONDERs and REWARD-NON-RESPONDERs subjectively rated their motivational levels as being higher when performing the Reward condition compared to the No-reward condition.

The scores from each of the $\mathrm{C}, \mathrm{S}$ and I versions of the AES were compared with paired samples t-tests (2 patients did not have informant AES). The clinician AES was significantly greater than the self-rated AES $\left(C\right.$ mean $=39.3, S$ mean $\left.=33.0, t_{(8)}=2.64, p=0.03\right)$, and there was a near-significant difference between the informant AES and the self-rated AES (I mean $=42.0, \mathrm{~S}$ mean $\left.=34.7, \mathrm{t}_{(6)}=2.43, \mathrm{p}=0.051\right)$. There was no difference between the clinician and informant AES $\left(C\right.$ mean $=38.6, I$ mean $\left.=42.0, t_{(6)}=-1.01, p=0.35\right)$. The scores for the reward responsiveness subscale of the BAS scale (BAS-RR) negatively correlated (Pearson's correlation) with the self-rated apathy scores $\left(r_{(9)}=-0.958, p<0.005\right)$ but not with clinician or informant scores. However, an independent samples t-test comparing REWARDRESPONDER with REWARD-NON-RESPONDER patients revealed no significant 
differences between the groups for any of the apathy scores (clinician $\left(t_{(7)}=0.735, p=0.49\right.$ ), informant $\left(t_{(5)}=0.507, p=0.63\right)$ or self-rated $\left.\left(t_{(7)}=0.745, p=0.48\right)\right)$ or the mean BAS-RR scores $\left(t_{(7)}=0.86, p=0.42\right)$.

\section{Lesion Anatomy}

Figure 4 shows the lesion overlap images for the 4 REWARD-RESPONDER patients (panel A) and 5 REWARD-NON-RESPONDERs patients (panel B), with lesion subtraction (REWARD-NON-RESPONDERs minus REWARD-RESPONDERs) shown in Panel C. We assessed whether the striatum was more likely to be damaged in the REWARD-NONRESPONDERs than the REWARD-RESPONDERs by examining the proportion of the dorsal striatum $\mathrm{ROI}(\mathrm{R}$ ant. caudate $+\mathrm{R}$ ant. putamen) that was damaged in each group. This showed that voxels in the dorsal striatum ROI were more likely to be damaged in REWARDNON-RESPONDERs than in REWARD-RESPONDERs $(t=2.43, p=0.046)$. Exploratory analysis across all other brain regions showed that no other ROls were significantly more affected in the REWARD-NON-RESPONDERs than in the REWARD-RESPONDER group (threshold of $p<0.05$ uncorrected).

\section{Discussion}

In this randomised, double-blind, placebo-controlled proof-of-concept study, we investigated the interaction between dopaminergic sensitivity and response to incentive motivation in spatial neglect.

\section{Reward Effects on Cancellation Task Performance}

The presence of monetary incentive improved neglect as measured by overall performance on a cancellation task, which is consistent with previous findings and in line with the motivational effects of monetary reward on spatial attention reported in healthy adults 
(Anderson et al., 2011; Bagurdes, Mesulam, Gitelman, Weintraub, \& Small, 2008; Della Libera \& Chelazzi, 2006; Kiss et al., 2009; Lucas, Schwartz, et al., 2013; Malhotra et al., 2013; Small et al., 2005). Consistent with this, patients felt subjectively more motivated, as evidenced by the overall higher ratings on the VAS for the rewarded task.

\section{Effect of Levodopa on Cancellation Performance}

In the current study there was no overall effect of dopamine on cancellation performance at a group level. This is in contrast to other studies showing an improvement in neglect with dopamine agonists or Levodopa. The majority of reports have demonstrated a broadly positive effect (Fleet et al., 1987; Geminiani et al., 1998; Gorgoraptis et al., 2012; Hurford et al., 1998; Mukand et al., 2001), although two studies have shown a worsening of neglect with a single dose of a dopamine agonist (Barrett et al., 1999; Grujic et al., 1998). However, it should be noted that the first of these was a single-case study in an individual with motorintentional neglect. It has been suggested that only neglect patients with a specific pattern of neuroanatomical damage would be likely to respond to dopaminergic stimulation e.g., that patients with more extensive frontal damage would be least likely to improve. However, neuroanatomical analysis of dopamine responsiveness in neglect has not yet shown any systematic pattern (Gorgoraptis et al., 2012), although to our knowledge, no authors have yet examined whether striatal damage influences dopaminergic response. The current study was designed in order to examine whether such differential responses might be explained, at least to some extent, by the influence of motivational deficits and damage to reward circuitry. Therefore, we systematically assessed the interaction between levodopa treatment and reward responsiveness.

Interaction of Reward Response with Reward and Levodopa Effects on Cancellation Task Performance 
Across the whole patient group, there was no evidence for a synergistic effect of exogeneous dopamine administration with reward. It is possible that dopamine does not differentially influence performance on the two experimental task variants, but this is not supported by its distinct effects in REWARD-RESPONDERs and REWARD-NON-RESPONDERS (Figure 3C). Instead we would suggest that our results may be accounted for by inverted U-shape function associated with dopaminergic effects on cognitive performance (Cools \& D'Esposito, 2011). That is, optimal levels of brain dopamine are required to drive the modulation of neglect, but suboptimal or supraoptimal levels are likely to impair them (Figure 5). This is consistent with observed performance in the No-reward condition, where there was a suggestion that at a group level, L-dopa improved cancellation task performance compared to placebo.

Critically, this account is in keeping with the differential effects of L-Dopa in REWARDRESPONDERs versus REWARD-NON-RESPONDERs. The REWARD-RESPONDER group demonstrated no additional benefit of L-Dopa, but rather performance tended to deteriorate on the Reward task following a single L-Dopa dose. This finding would again support the inverted U-shape relationship as discussed above. It has been shown that incentive motivation leads to increased endogeneous dopaminergic activity (Wassum, Ostlund, Loewinger, \& Maidment, 2013), and it is therefore possible that the administration of exogenous dopamine could lead to excessive dopamine levels in the REWARDRESPONDER group when performing the pound coin condition after receiving L-Dopa. The tendency to worse performance on this condition also provides evidence against a ceiling effect preventing a synergistic response to reward and dopamine in this group. Moreover, it is consistent with an inverted U-shape function relating dopamine levels to cognitive performance (Cools \& D'Esposito, 2011). . The REWARD-NON-RESPONDER group, having previously showed no reward-attention interaction, demonstrated an overall improvement in performance on the Reward task following L-Dopa. This suggests that L- 
Dopa 'induces' a reward-attention interaction in the REWARD-NON-RESPONDER group, and is in keeping with the single-case study carried out by Adam and colleagues (2013). It is also consistent with Parkinson's Disease research where blunted reward processing in patients off medications was enhanced following dopamine agonist administration (Bodi et al., 2009). This induction of a reward-attention interaction might be explained by a variation in optimal levels of dopamine between the REWARD-RESPONDER and REWARD-NONRESPONDER groups secondary to lesion anatomy (see below).

\section{Anatomy of Reward-Responsiveness and the Interaction between Motivational and Attentional Networks}

The patient group had a wide range of lesions as previously described in the neglect literature (Lunven et al., 2015; Ramsey et al., 2016). However, as can be seen in Figure 4, and as per our ROI analysis, the REWARD-NON-RESPONDER group was more likely to have damage to the dorsal striatum (DS). This result was obtained with a relatively lenient threshold, and should be interpreted with some caution, but it is in keeping with prior studies, including our own (Malhotra et al., 2013). Below we offer an account that integrates the lesion findings in this context. While the DS is primarily associated with action selection and movement, there is considerable evidence for its role in motivational processing. For example PET studies have reported an increase in dopamine release in both DS and ventral striatum when participants played a video game for anticipated monetary incentives, similar to our paradigm (Koepp et al., 1998), and in DS when healthy humans performed card selection tasks for monetary gain (Zald et al., 2004). Similarly, fMRI studies have reported increases in blood oxygenation level dependent responses in DS in anticipation of monetary reward (Knutson, Adams, Fong, \& Hommer, 2001). Interestingly, there was no difference between groups in VAS score, with both groups rating their motivation to be higher for the Reward versus the No-reward condition. Thus, damage to DS in this and our previous study appeared to disrupt the behavioural sequelae of incentive motivation, even though 
REWARD-NON-RESPONDERs reported greater motivation for the Reward condition. This is in keeping with findings from healthy humans showing that the striatum is a key structure linking motivation to action (Harsay et al., 2011).

The observation that the REWARD-NON-RESPONDERs were more likely to have damage to the right dorsal striatum is also consistent with animal studies demonstrating that neglectlike behaviour caused by cortical lesions is induced or worsened by additional striatal damage (Carli, Jones, \& Robbins, 1989; Christakou, Robbins, \& Everitt, 2005; Van Vleet, Heldt, Pyter, Corwin, \& Reep, 2003), and it should be noted that the majority of animal experiments evaluating attention explicitly probe reward-seeking behaviour. Moreover, such neglect-like symptoms in animals can be modulated by dopaminergic stimulation (van Vleet, Heldt, Corwin, \& Reep, 2003). Neuroanatomical studies of clinical neglect have understandably tended to focus on frontoparietal networks involved in spatial and non-spatial aspects of attention, but it is important to consider how the syndrome might result from interactions between component deficits (Rafal, 1994). Although some studies have investigated neglect solely secondary to basal ganglia damage, there have been very few clinical studies exploring the effects of damage to reward-related striatal systems in combination with fronto-parietal network disruption, even though this is likely to result in altered interactions between motivational and attentional systems (Bourgeois, Chelazzi, \& Vuilleumier, 2016).

Our findings do not rule out the possibility that the effects of both reward and L-dopa are mediated by an increase in arousal. In fact, we have previously found that incentive motivation does appear to affect arousal on a trial-by-trial basis (Olgiati et al., 2016). In addition, the apparent absence of hemispace-specific responses to reward and dopaminergic stimulation, implies that they both might act via a non-lateralised attentional mechanism. However, the authors of two previous studies have concluded that the positive effects of 
dopaminergic stimulation in neglect are unlikely to be mediated by arousal (Geminiani et al., 1998; Gorgoraptis et al., 2012). Perhaps crucially, L-dopa, which has clearly been shown to modulate arousal in Parkinson's Disease (Horvath \& Meares, 1974), was not the compound used in those studies. When L-dopa has been found to improve neglect, a boost in arousal has been suggested as a possible mechanism of action (Mukand et al., 2001).

\section{Relationship of Apathy to Reward Response}

The presence of apathy has recently been associated with reward insensitivity in patients with ischaemic brain damage (Adam et al., 2013; Rochat et al., 2013; Schmidt et al., 2008). In our study, the self-reported apathy scores of patients showed a strong negative correlation with their scores on the reward responsiveness subscale of the BAS scale, with the more apathetic patients being less responsive to reward on this questionnaire. This is likely to relate to both these scales being self-rated, and the relative similarity of questions in each. In contrast, clinician and informant scores did not correlate with the BAS-RR scale, suggesting that there was not a clear relationship between apathy and reward responsiveness in this group. Moreover, there was no significant difference in any of the apathy scores between the REWARD-RESPONDER and REWARD-NON-RESPONDER groups. It is possible that the reported differences between apathy and behavioural reward responsiveness may reflect differences in how apathy has been defined across other studies. Whereas Adam et al. (2013) and Rochat and colleagues (Rochat et al., 2013) measured apathy using the Apathy Inventory, the present study utilised instead the AES, the latter considered to be one of the most psychometrically robust broad measures of apathy (Clarke et al., 2011). The absence of a relationship between apathy and lack of reward response as seen in the current study is consistent with the results of Chong and colleagues (Chong et al., 2015), who demonstrated that even in the absence of apathy, PD patients showed deficits in reward-incentivised decision-making. 


\section{Dopaminergic Treatment in Stroke}

In addition to being used in trials attempting to improve neglect, it has been suggested that dopaminergic stimulation may improve other post-stroke cognitive deficits such as aphasia, or augment stroke rehabilitation as a whole (Bhakta et al., 2014; Gill \& Leff, 2012). However, to-date there have been no universally successful trials of dopaminergic stimulation in stroke rehabilitation, and the results of the current study suggest that assessing motivational responsiveness may be helpful in determining which individuals will respond to L-dopa or dopamine agonists. As discussed above, apathy has been shown by some groups to relate to reward responsiveness, and has been shown to improve with dopaminergic stimulation (Adam et al., 2013; Kohno et al., 2010). It might be that subgroup analysis assessing apathy status could help identify those patients who are more likely to respond. However, we note that in our group of patients, apathy did not directly map into blunted reward response, and apathy scales may not be as sensitive as the direct assessment of reward sensitivity.

\section{Conclusions}

The current study demonstrates that there is an interaction between dopamine responsiveness and sensitivity to incentive motivation in patients with spatial neglect, which may be mediated by striatal damage. Reward improved performance across the patient group, and a single dose of L-dopa only improved reward-related performance in those individuals who did not previously respond to reward. These results are consistent with the proposal that the relationship between dopamine and neglect is dose-dependent, and dictated by an inverted $U$-shape function. These results demonstrate the importance of the interactions between motivational and attentional deficits for our understanding of responsiveness to dopaminergic stimulation in neglect, and potentially in the wider field of stroke rehabilitation. 


\section{Acknowledgements}

This study was directly supported by the Biomedical Research Centre at Imperial College London and a HEFCE Clinical Senior Lectureship Award to PM. D.S. acknowledges support from the Spanish Ministry of Economy and Competitiveness (MINECO), through the 'Severo Ochoa' Programme for Centres/Units of Excellence in R\&D (SEV-2015-490) and project grants PSI2016-76443-P from MINECO and PI-2017-25 from the Basque Government.

\section{Author Contributions}

$\mathrm{KL}, \mathrm{DS}, \mathrm{GB}, \mathrm{CR}$ \& PM conceived and designed the study. $\mathrm{KL}, \mathrm{PB}$ and PM analysed the data.

The manuscript was written by $\mathrm{KL}, \mathrm{PB}, \mathrm{CR}, \mathrm{OH}, \mathrm{AN}$ and $\mathrm{PM}$.

\section{Potential Competing Interests}

The authors report no potential conflicts of interest. 


\section{References}

Adam, R., Leff, A., Sinha, N., Turner, C., Bays, P., Draganski, B., \& Husain, M. (2013). Dopamine reverses reward insensitivity in apathy following globus pallidus lesions. Cortex, 49(5), 1292-1303. doi: 10.1016/j.cortex.2012.04.013

Anderson, B. A., Laurent, P. A., \& Yantis, S. (2011). Value-driven attentional capture. Proc Natl Acad Sci U S A, 108(25), 10367-10371. doi: 1104047108 [pii]

Bagurdes, L. A., Mesulam, M. M., Gitelman, D. R., Weintraub, S., \& Small, D. M. (2008). Modulation of the spatial attention network by incentives in healthy aging and mild cognitive impairment. Neuropsychologia, 46(12), 2943-2948. doi: S00283932(08)00257-1 [pii]

Barrett, A. M., Crucian, G. P., Schwartz, R. L., \& Heilman, K. M. (1999). Adverse effect of dopamine agonist therapy in a patient with motor-intentional neglect. Arch Phys Med Rehabil, 80(5), 600-603.

Bhakta, B. B., Hartley, S., Holloway, I., Couzens, J. A., Ford, G. A., Meads, D., . . Farrin, A. J. (2014). The DARS (Dopamine Augmented Rehabilitation in Stroke) trial: protocol for a randomised controlled trial of Co-careldopa treatment in addition to routine NHS occupational and physical therapy after stroke. Trials, 15, 316. doi: 10.1186/17456215-15-316

Bodi, N., Keri, S., Nagy, H., Moustafa, A., Myers, C. E., Daw, N., . . Gluck, M. A. (2009). Reward-learning and the novelty-seeking personality: a between- and within-subjects study of the effects of dopamine agonists on young Parkinson's patients. Brain, 132(Pt 9), 2385-2395.

Bourgeois, A., Chelazzi, L., \& Vuilleumier, P. (2016). How motivation and reward learning modulate selective attention. Prog Brain Res, 229, 325-342. doi: 10.1016/bs.pbr.2016.06.004

Bourgeois, A., Neveu, R., Bayle, D. J., \& Vuilleumier, P. (2017). How does reward compete with goal-directed and stimulus-driven shifts of attention? Cogn Emot, 31(1), 109-118. doi: 10.1080/02699931.2015.1085366

Bowen, A., Hazelton, C., Pollock, A., \& Lincoln, N. B. (2013). Cognitive rehabilitation for spatial neglect following stroke. Cochrane Database Syst Rev(7), CD003586. doi: 10.1002/14651858.CD003586.pub3 
Cappa, S., Sterzi, R., Vallar, G., \& Bisiach, E. (1987). Remission of hemineglect and anosognosia during vestibular stimulation. Neuropsychologia, 25(5), 775-782.

Carli, M., Jones, G. H., \& Robbins, T. W. (1989). Effects of unilateral dorsal and ventral striatal dopamine depletion on visual neglect in the rat: a neural and behavioural analysis. [Research Support, Non-U.S. Gov't]. Neuroscience, 29(2), 309-327.

Carver, C. S., \& White, T. L. (1994). Behavioral Inhibition, Behavioral Activation, and Affective Responses to Impending Reward and Punishment: The BIS/BAS Scales. $J$ Pers Soc Psychol, 67(2), 319-333.

Chong, T. T., Bonnelle, V., Manohar, S., Veromann, K. R., Muhammed, K., Tofaris, G. K., . . . Husain, M. (2015). Dopamine enhances willingness to exert effort for reward in Parkinson's disease. Cortex, 69, 40-46. doi: 10.1016/j.cortex.2015.04.003

Chong, T. T., \& Husain, M. (2016). The role of dopamine in the pathophysiology and treatment of apathy. Prog Brain Res, 229, 389-426. doi: 10.1016/bs.pbr.2016.05.007

Christakou, A., Robbins, T. W., \& Everitt, B. J. (2005). Prolonged neglect following unilateral disruption of a prefrontal cortical-dorsal striatal system. Eur J Neurosci, 21(3), 782792. doi: EJN3892 [pii]

Clarke, D. E., Ko, J. Y., Kuhl, E. A., van Reekum, R., Salvador, R., \& Marin, R. S. (2011). Are the available apathy measures reliable and valid? A review of the psychometric evidence. [Research Support, N.I.H., Extramural

Review]. J Psychosom Res, 70(1), 73-97. doi: 10.1016/j.jpsychores.2010.01.012

Cools, R., \& D'Esposito, M. (2011). Inverted-U-shaped dopamine actions on human working memory and cognitive control. Biol Psychiatry, 69(12), e113-125. doi: 10.1016/j.biopsych.2011.03.028

Corwin, J. V., Kanter, S., Watson, R. T., Heilman, K. M., Valenstein, E., \& Hashimoto, A. (1986). Apomorphine has a therapeutic effect on neglect produced by unilateral dorsomedial prefrontal cortex lesions in rats. Exp Neurol, 94(3), 683-698.

Czernecki, V., Pillon, B., Houeto, J. L., Pochon, J. B., Levy, R., \& Dubois, B. (2002). Motivation, reward, and Parkinson's disease: influence of dopatherapy. Neuropsychologia, 40(13), 2257-2267.

Dalmaijer, E. S., Li, K. M. S., Gorgoraptis, N., Leff, A. P., Cohen, D. L., Parton, A. D., . . . Malhotra, P. A. (2018). Randomised, double-blind, placebo-controlled crossover study of single-dose guanfacine in unilateral neglect following stroke. J Neurol Neurosurg Psychiatry. doi: 10.1136/jnnp-2017-317338

Della Libera, C., \& Chelazzi, L. (2006). Visual selective attention and the effects of monetary rewards. Psychol Sci, 17(3), 222-227. doi: PSCI1689 [pii] 
Desikan, R. S., Segonne, F., Fischl, B., Quinn, B. T., Dickerson, B. C., Blacker, D., . . . Killiany, R. J. (2006). An automated labeling system for subdividing the human cerebral cortex on MRI scans into gyral based regions of interest. Neuroimage, 31(3), 968-980. doi: 10.1016/j.neuroimage.2006.01.021

Fleet, W. S., Valenstein, E., Watson, R. T., \& Heilman, K. M. (1987). Dopamine agonist therapy for neglect in humans. Neurology, 37, 1765-1771.

Gajardo-Vidal, A., Lorca-Puls, D. L., Crinion, J. T., White, J., Seghier, M. L., Leff, A. P., . . . Price, C. J. (2018). How distributed processing produces false negatives in voxelbased lesion-deficit analyses. Neuropsychologia, 115, 124-133. doi: 10.1016/j.neuropsychologia.2018.02.025

Geminiani, G., Bottini, G., \& Sterzi, R. (1998). Dopaminergic stimulation in unilateral neglect. J Neurol Neurosurg Psychiatry, 65(3), 344-347.

Gill, S. K., \& Leff, A. P. (2012). Dopaminergic therapy in aphasia. Aphasiology, 28(2), 155170. doi: $10.1080 / 02687038.2013 .802286$

Gorgoraptis, N., Mah, Y. H., Machner, B., Singh-Curry, V., Malhotra, P., Hadji-Michael, M., . . . Husain, M. (2012). The effects of the dopamine agonist rotigotine on hemispatial neglect following stroke. Brain, 135(Pt 8), 2478-2491. doi: 10.1093/brain/aws154

Grujic, Z., Mapstone, M., Gitelman, D. R., Johnson, N., Weintraub, S., Hays, A., . . . Mesulam, M. M. (1998). Dopamine agonists reorient visual exploration away from the neglected hemispace. Neurology, 51(5), 1395-1398.

Harsay, H. A., Cohen, M. X., Oosterhof, N. N., Forstmann, B. U., Mars, R. B., \& Ridderinkhof, K. R. (2011). Functional connectivity of the striatum links motivation to action control in humans. J Neurosci, 31(29), 10701-10711. doi: 31/29/10701 [pii]

Horvath, T. B., \& Meares, R. A. (1974). L-dopa and arousal. J Neurol Neurosurg Psychiatry, 37(4), 416-420.

Hurford, P., Stringer, A. Y., \& Jann, B. (1998). Neuropharmacologic treatment of hemineglect: a case report comparing bromocriptine and methylphenidate. Arch Phys Med Rehabil, 79(3), 346-349.

Kiss, M., Driver, J., \& Eimer, M. (2009). Reward priority of visual target singletons modulates event-related potential signatures of attentional selection. Psychol Sci, 20(2), 245251. doi: PSCI2281 [pii]

10.1111/j.1467-9280.2009.02281.x

Knutson, B., Adams, C. M., Fong, G. W., \& Hommer, D. (2001). Anticipation of increasing monetary reward selectively recruits nucleus accumbens. [Clinical Trial

Research Support, U.S. Gov't, P.H.S.]. The Journal of Neuroscience, 21(16), RC159. 
Koch, G., Bonni, S., Giacobbe, V., Bucchi, G., Basile, B., Lupo, F., . . . Caltagirone, C. (2012). Theta-burst stimulation of the left hemisphere accelerates recovery of hemispatial neglect. [Research Support, Non-U.S. Gov't]. Neurology, 78(1), 24-30. doi: 10.1212/WNL.0b013e31823ed08f

Koepp, M. J., Gunn, R. N., Lawrence, A. D., Cunningham, V. J., Dagher, A., Jones, T., . . . Grasby, P. M. (1998). Evidence for striatal dopamine release during a video game. Nature, 393(6682), 266-268. doi: 10.1038/30498

Kohno, N., Abe, S., Toyoda, G., Oguro, H., Bokura, H., \& Yamaguchi, S. (2010). Successful treatment of post-stroke apathy by the dopamine receptor agonist ropinirole. $J$ Clin Neurosci, 17(6), 804-806. doi: 10.1016/j.jocn.2009.09.043

Lecce, F., Rotondaro, F., Bonni, S., Carlesimo, A., Thiebaut de Schotten, M., Tomaiuolo, F., \& Doricchi, F. (2015). Cingulate neglect in humans: disruption of contralesional reward learning in right brain damage. Cortex, 62, 73-88. doi: 10.1016/j.cortex.2014.08.008

Li, K., Russell, C., Balaji, N., Saleh, Y., Soto, D., \& Malhotra, P. A. (2016). The effects of motivational reward on the pathological attentional blink following right hemisphere stroke. Neuropsychologia, 92, 190-196. doi: 10.1016/j.neuropsychologia.2016.03.037

Lucas, N., Saj, A., Schwartz, S., Ptak, R., Thomas, C., Conne, P., . . Vuilleumier, P. (2013). Effects of pro-cholinergic treatment in patients suffering from spatial neglect. Front Hum Neurosci, 7, 574. doi: 10.3389/fnhum.2013.00574

Lucas, N., Schwartz, S., Leroy, R., Pavin, S., Diserens, K., \& Vuilleumier, P. (2013). Gambling against neglect: unconscious spatial biases induced by reward reinforcement in healthy people and brain-damaged patients. Cortex, 49(10), 26162627. doi: 10.1016/j.cortex.2013.06.004

Lunven, M., Thiebaut De Schotten, M., Bourlon, C., Duret, C., Migliaccio, R., Rode, G., \& Bartolomeo, P. (2015). White matter lesional predictors of chronic visual neglect: a longitudinal study. Brain. doi: 10.1093/brain/awu389

Malhotra, P. A., Soto, D., Li, K., \& Russell, C. (2013). Reward modulates spatial neglect. J Neurol Neurosurg Psychiatry, 84(4), 366-369. doi: 10.1136/jnnp-2012-303169

Marin, R. S., Biedrzycki, R. C., \& Firinciogullari, S. (1991). Reliability and validity of the Apathy Evaluation Scale. Psychiatry Res, 38(2), 143-162.

Marshall, J. F., \& Gotthelf, T. (1979). Sensory inattention in rats with 6-hydroxydopamineinduced degeneration of ascending dopaminergic neurons: apomorphine-induced reversal of deficits. Exp Neurol, 65(2), 398-411.

Mesulam, M. M. (1985). Principles of behavioral neurology. Philadelphia: F.A. Davis.

Muhammed, K., Manohar, S., Ben Yehuda, M., Chong, T. T., Tofaris, G., Lennox, G., . . . Husain, M. (2016). Reward sensitivity deficits modulated by dopamine are associated 
with apathy in Parkinson's disease. Brain, 139(Pt 10), 2706-2721. doi: 10.1093/brain/aww188

Mukand, J. A., Guilmette, T. J., Allen, D. G., Brown, L. K., Brown, S. L., Tober, K. L., \& Vandyck, W. R. (2001). Dopaminergic therapy with carbidopa L-dopa for left neglect after stroke: a case series. Arch Phys Med Rehabil, 82(9), 1279-1282.

O'Shea, J., Revol, P., Cousijn, H., Near, J., Petitet, P., Jacquin-Courtois, S., . . Rossetti, Y. (2017). Induced sensorimotor cortex plasticity remediates chronic treatment-resistant visual neglect. Elife, 6. doi: 10.7554/eLife.26602

Olgiati, E., Russell, C., Soto, D., \& Malhotra, P. (2016). Motivation and attention following hemispheric stroke. Prog Brain Res, 229, 343-366. doi: 10.1016/bs.pbr.2016.06.011

Pierce, S. R., \& Buxbaum, L. J. (2002). Treatments of unilateral neglect: a review. Arch Phys Med Rehabil, 83(2), 256-268.

Pizzamiglio, L., Fasotti, L., Jehkonen, M., Antonucci, G., Magnotti, L., Boelen, D., \& Asa, S. (2004). The use of optokinetic stimulation in rehabilitation of the hemineglect disorder. Cortex, 40(3), 441-450.

Rafal, R. D. (1994). Neglect. Current Opinion in Neurobiology, 4, 231-236.

Ramsey, L. E., Siegel, J. S., Baldassarre, A., Metcalf, N. V., Zinn, K., Shulman, G. L., \& Corbetta, M. (2016). Normalization of network connectivity in hemispatial neglect recovery. Ann Neurol, 80(1), 127-141. doi: 10.1002/ana.24690

Rinne, P., Hassan, M., Goniotakis, D., Chohan, K., Sharma, P., Langdon, D., . . Bentley, P. (2013). Triple dissociation of attention networks in stroke according to lesion location. Neurology, 81(9), 812-820. doi: 10.1212/WNL.0b013e3182a2ca34

Robertson, I. H. (2012). The neglected role of reward in rehabilitation. J Neurol Neurosurg Psychiatry. doi: 10.1136/jnnp-2012-303547

Rochat, L., Van der Linden, M., Renaud, O., Epiney, J. B., Michel, P., Sztajzel, R., . . . Annoni, J. M. (2013). Poor reward sensitivity and apathy after stroke: implication of basal ganglia. Neurology, 81(19), 1674-1680. doi: 10.1212/01.wnl.0000435290.49598.1d

Rossetti, Y., Rode, G., Pisella, L., Farne, A., Li, L., Boisson, D., \& Perenin, M. T. (1998). Prism adaptation to a rightward optical deviation rehabilitates left hemispatial neglect. Nature, 395(6698), 166-169.

Schmidt, L., d'Arc, B. F., Lafargue, G., Galanaud, D., Czernecki, V., Grabli, D., . . . Pessiglione, M. (2008). Disconnecting force from money: effects of basal ganglia damage on incentive motivation. Brain, 131(Pt 5), 1303-1310.

Schultz, W. (1998). Predictive reward signal of dopamine neurons. J Neurophysiol, 80(1), 127. 
Shore, D. M., Rafal, R., \& Parkinson, J. A. (2011). Appetitive motivational deficits in individuals with Parkinson's disease. Mov Disord, 26(10), 1887-1892. doi: $10.1002 / \mathrm{mds} .23736$

Small, D. M., Gitelman, D., Simmons, K., Bloise, S. M., Parrish, T., \& Mesulam, M. M. (2005). Monetary incentives enhance processing in brain regions mediating top-down control of attention. Cereb Cortex, 15(12), 1855-1865.

van der Kemp, J., Dorresteijn, M., Ten Brink, A. F., Nijboer, T. C., \& Visser-Meily, J. M. (2017). Pharmacological Treatment of Visuospatial Neglect: a Systematic Review. J Stroke Cerebrovasc Dis. doi: 10.1016/j.jstrokecerebrovasdis.2017.02.012

van Vleet, T. M., Heldt, S. A., Corwin, J. V., \& Reep, R. L. (2003). Infusion of apomorphine into the dorsocentral striatum produces acute drug-induced recovery from neglect produced by unilateral medial agranular cortex lesions in rats. Behav Brain Res, 143(2), 147-157.

Van Vleet, T. M., Heldt, S. A., Pyter, B., Corwin, J. V., \& Reep, R. L. (2003). Effects of light deprivation on recovery from neglect and extinction induced by unilateral lesions of the medial agranular cortex and dorsocentral striatum. Behav Brain Res, 138(2), 165178.

Wassum, K. M., Ostlund, S. B., Loewinger, G. C., \& Maidment, N. T. (2013). Phasic mesolimbic dopamine release tracks reward seeking during expression of Pavlovianto-instrumental transfer. Biol Psychiatry, 73(8), 747-755. doi: 10.1016/j.biopsych.2012.12.005

Weintraub, S., \& Mesulam, M. M. (1988). Visual hemispatial inattention: stimulus parameters and exploratory strategies. J Neurol Neurosurg Psychiatry, 51(12), 1481-1488.

Wilson, B., Cockburn, J., \& Halligan, P. W. (1987). Behavioural inattention test. Bury St. Edmunds: Thames Valley.

Zald, D. H., Boileau, I., El-Dearedy, W., Gunn, R., McGlone, F., Dichter, G. S., \& Dagher, A. (2004). Dopamine transmission in the human striatum during monetary reward tasks. J Neurosci, 24(17), 4105-4112. doi: 10.1523/JNEUROSCI.4643-03.2004

Zhang, Y., Zhang, J., Oishi, K., Faria, A. V., Jiang, H., Li, X., . . Mori, S. (2010). Atlasguided tract reconstruction for automated and comprehensive examination of the white matter anatomy. Neuroimage, 52(4), 1289-1301. doi: 10.1016/j.neuroimage.2010.05.049 


\section{Figure Legends}

\section{Figure 1}

\section{Study Design}

Schematic showing randomised, single-dose, placebo-controlled crossover study design

\section{Figure 2}

\section{Cancellation task from Malhotra et al. (2013)}

In each array there were 54 targets (27 each side of midline) amongst 52 matched distractors; targets and distractors were $12 \mathrm{~mm}$ in diameter. Distractors were produced for each task by using a Gaussian blur to merge the features of each target until they were no longer distinguishable but remained identical in overall distribution of hue and luminance (for further details see Malhotra et al. (2013)). In order to ensure that participants did not learn exactly where targets were on the display from session to session, we employed 5 different arrays. Each of these employed the same number of targets and distractors, and the same configurations were used for both conditions in each individual session. Condition order was counterbalanced within and between individuals.

\section{A: Reward condition}

For the Reward condition, patients were asked to find and mark all the target images of pound coins on the A3 sized array, and were informed that they would receive one pound for each target that they correctly marked.

\section{B: No-reward condition}

For the No-reward condition, patients were asked to find and mark all the images of buttons on the A3 sheet of paper and were informed that they would not receive any money in relation to their performance.

\section{Figure 3:}

\section{Effect of Reward and L-Dopa on Cancellation Performance at a Group Level}

\section{A: Overall effect of reward on neglect on cancellation tasks across all sessions}


Log-transformed reverse scores for the total number of targets found. Values closer to $0=$ greater number of targets found; There was a main effect of condition $\left(F_{(1,8)}=7.89, p<0.05\right)$, with more targets found in the Reward condition than the No-reward condition Error bars = standard error.

\section{B: Effect of L-Dopa versus placebo on No-Reward and Reward conditions}

Difference between performance post-L-Dopa and performance post-placebo in both conditions expressed as a percentage. L-dopa led to a $13 \%$ increase in targets found on the No-reward task whereas performance on the Reward task worsened by approximately $10 \%$ after L-dopa when compared to placebo. Error bars = standard error.

C: Change in task performance with L-Dopa for REWARD-RESPONDERs and REWARD-NON-RESPONDERs on both task conditions

Effect of L-Dopa on performance (post-L-Dopa minus pre-L-Dopa) on the Reward and Noreward tasks for the two groups. REWARD-NON-RESPONDERs $(n=4)$ improved by $44 \%$ whereas REWARD-RESPONDERs' $(n=5)$ performance worsened by $13 \%$ on the Reward task. The two groups did not show significantly different responses to L-dopa in the Noreward condition. Error bars $=$ standard error, ${ }^{*} p<0.05$.

Figure 4: Anatomical differences between REWARD-RESPONDERs \& REWARD-NONRESPONDERS

A: Lesion overlap images for 4 REWARD-NON-RESPONDER patients; B: Lesion overlap images for 5 REWARD-RESPONDER patients; C: Lesion subtraction showing regions damaged in REWARD-NON-RESPONDERs that were less likely to be damaged in reward responders.

Sagittal images show slice levels. For panels $A \& B$, the number of patients with damage to each region is represented by the multi-coloured bars, with one patient depicted by the leftward-most violet colour, and the maximum number of patients as indicated by red on the 
far right. In panel C, regions that were damaged in REWARD-NON-RESPONDERs that were less likely to be damaged in REWARD-RESPONDERs, represented as a percentage as indicated by the legend to the right of the panel.

\section{Figure 5: Dose-dependent effects of Dopamine on cancellation task performance}

A: We propose that the relationship between cancellation task performance and dopamine levels follows an inverted U-shape function (Cools \& D'Esposito, 2011), where both insufficient (for example, in the absence of both reward and levodopa) and excessive (for example, the administration of levodopa with reward) dopamine levels lead to worse performance. $\mathrm{NR}=$ No-reward; $\mathrm{R}=$ Reward

\section{B: Dose-dependent effects of dopamine on cancellation task performance according to reward response}

Optimal levels of dopamine may vary between individuals who demonstrate an initial rewardattention response (REWARD-RESPONDERs, RRs = red curve) and those who do not (REWARD-NON-RESPONDERs, RNRs = green curve), which may, in part, be dictated by basal dopamine concentrations. Thus, for the same rise in dopamine level (black arrow), the performance of REWARD-NON-RESPONDERs may improve (green curve) whereas that of REWARD-RESPONDERs deteriorates (red curve). Furthermore, REWARD-NONRESPONDERs may be operating within a wider range, requiring greater concentrations to effect a similar change in performance to REWARD-RESPONDERs. 


\begin{tabular}{|c|c|c|c|c|c|c|c|c|c|}
\hline \multirow{2}{*}{ Patient } & \multirow{2}{*}{ Sex } & \multirow{2}{*}{$\begin{array}{c}\text { Age } \\
\text { (years) }\end{array}$} & \multirow{2}{*}{$\begin{array}{c}\text { Time since Stroke } \\
\text { (months) }\end{array}$} & \multicolumn{3}{|c|}{ BIT Star Cancellation } & \multicolumn{3}{|c|}{ Mesulam Shape Cancellation } \\
\hline & & & & L & $\mathbf{R}$ & Total (54) & L & $\mathbf{R}$ & Total $(60)$ \\
\hline 1 & M & 74 & 15 & 0 & 5 & 5 & 0 & 2 & 2 \\
\hline 2 & M & 49 & 87 & 0 & 25 & 25 & 0 & 25 & 25 \\
\hline 3 & M & 64 & 70 & 21 & 27 & 48 & 17 & 27 & 44 \\
\hline 4 & M & 59 & 30 & 20 & 20 & 40 & 17 & 18 & 35 \\
\hline 5 & M & 70 & 45 & 23 & 27 & 50 & 22 & 24 & 46 \\
\hline 6 & M & 84 & 8 & 8 & 13 & 21 & 5 & 18 & 23 \\
\hline 7 & M & 59 & 2 & 3 & 22 & 25 & 0 & 15 & 15 \\
\hline 8 & $\mathrm{~F}$ & 59 & 3 & 0 & 17 & 17 & 0 & 15 & 15 \\
\hline 9 & M & 64 & 4 & 0 & 15 & 15 & 0 & 10 & 10 \\
\hline
\end{tabular}

Table 1: Patient Demographics and Performance on Standard Cancellation Tasks

All patients presented following their first stroke and apart from Patient 8 , all had ischaemic strokes. All patients had manifested clinical neglect at presentation. BIT Star and Mesulam Shape Cancellation scores indicate number of targets found in each hemifield as well as total number of targets found. All patients commenced cancellation on the right side of the array. 


\begin{tabular}{|c|c|c|c|c|c|c|c|c|c|c|}
\hline \multirow{2}{*}{ Patient } & \multicolumn{3}{|c|}{ AES Scores } & \multicolumn{4}{|c|}{ BIS/BAS Scores } & \multicolumn{2}{|c|}{ Mean VAS Scores } & \multirow{2}{*}{$\begin{array}{l}\text { Reward- } \\
\text { Attention } \\
\text { Response }\end{array}$} \\
\hline & C & I & $\mathbf{s}$ & BAS-Drive & BAS-Fun & BAS-RR & BIS & NR & $\mathbf{R}$ & \\
\hline 1 & 30 & 34 & 34 & 10 & 10 & 18 & 24 & 62.8 & 72.8 & RNR \\
\hline 2 & 24 & 24 & 19 & 13 & 14 & 20 & 23 & 37.8 & 69.3 & $\mathrm{RR}$ \\
\hline 3 & 25 & 43 & 27 & 14 & 10 & 19 & 8 & 35.3 & 83.5 & RNR \\
\hline 4 & 49 & 60 & 41 & 14 & 12 & 17 & 23 & 46.8 & 97.3 & $\mathrm{RR}$ \\
\hline 5 & 55 & 49 & 52 & 12 & 9 & 14 & 19 & 90.0 & 90.0 & $\mathrm{RR}$ \\
\hline 6 & 42 & - & 28 & 16 & 10 & 20 & 26 & 71.0 & 87.0 & $\mathrm{RR}$ \\
\hline 7 & 39 & 43 & 36 & 11 & 13 & 17 & 26 & 76.8 & 75.5 & $\mathrm{RR}$ \\
\hline 8 & 48 & 41 & 34 & 14 & 14 & 18 & 24 & 26.3 & 45.8 & RNR \\
\hline 9 & 42 & - & 26 & 15 & 15 & 20 & 23 & 79.0 & 100.0 & RNR \\
\hline
\end{tabular}

\section{Table 2: Apathy and Motivation Scores}

$\mathrm{C}=$ clinician, $\mathrm{I}=$ informant $\mathrm{S}=$ self-rated versions of the AES = apathy evaluation scale; $\mathrm{BIS} / \mathrm{BAS}=$ behavioural inhibition/approach system; BAS-RR = reward responsiveness subscale of the BAS; VAS = visual analogue scale; $N R=$ No-reward condition; $R=$ Reward condition; $R R=$ REWARDRESPONDERs; RNR = REWARD-NON-RESPONDERs 


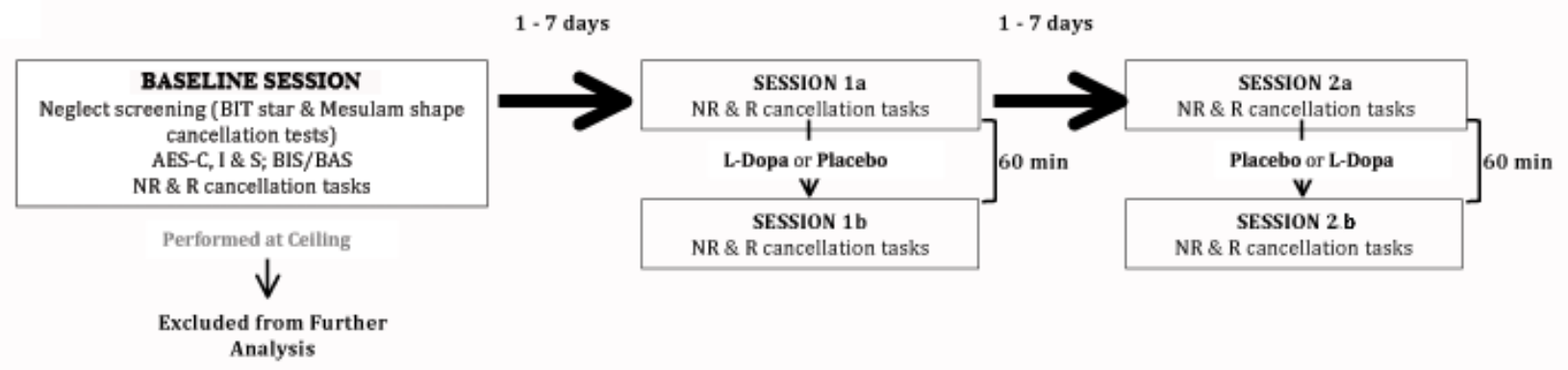

Figure 1 
A
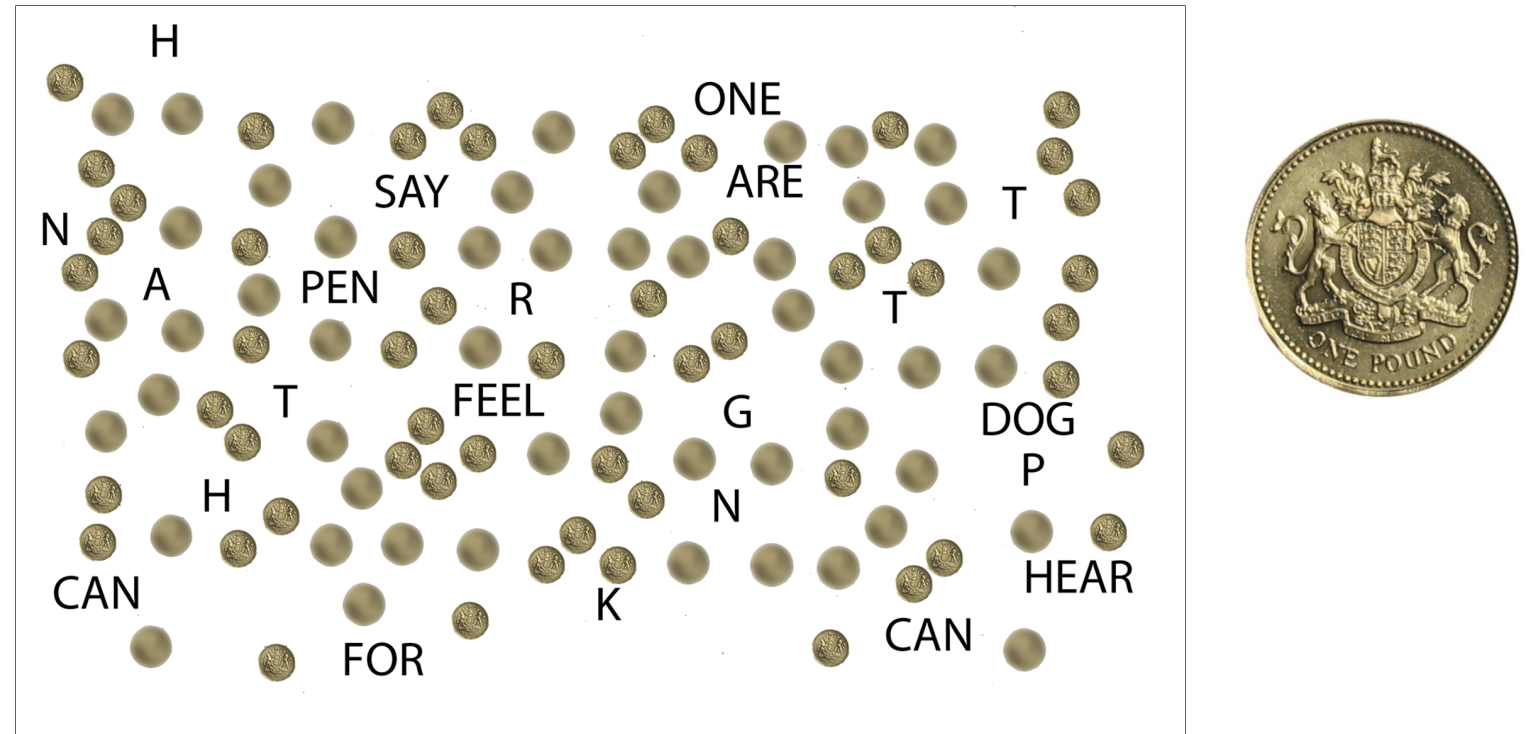

B

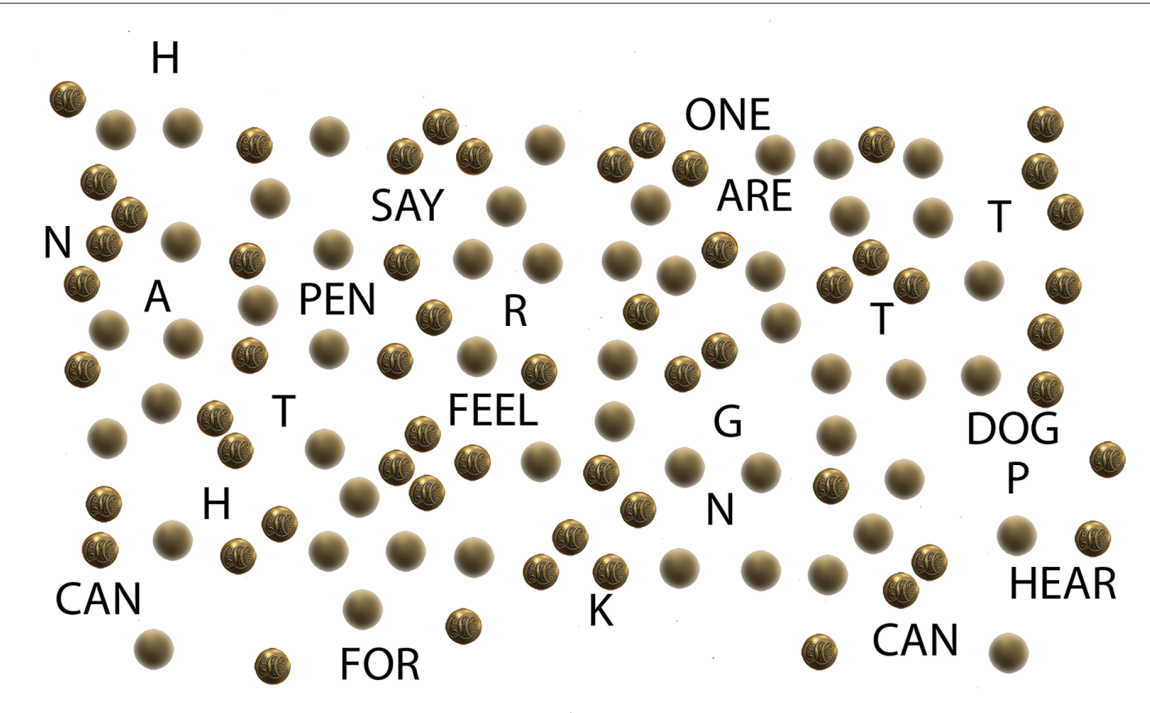

Figure 2 
A

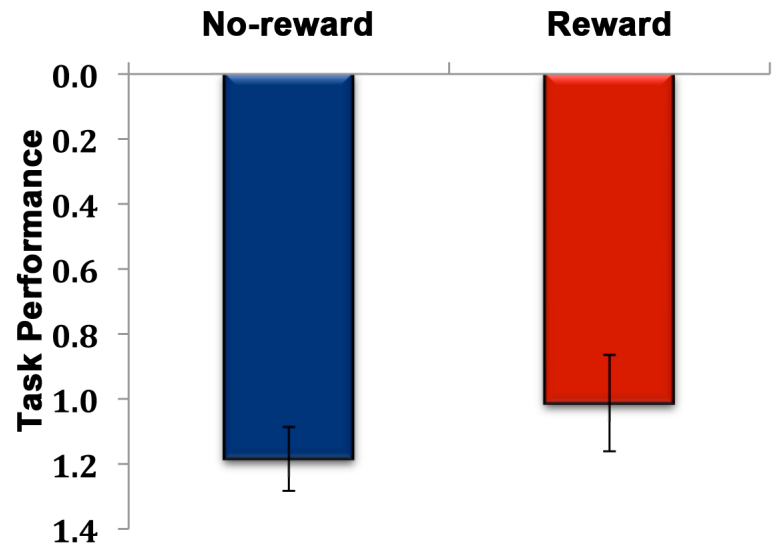

C

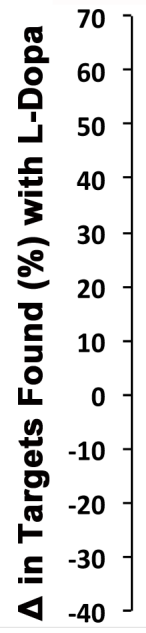

B

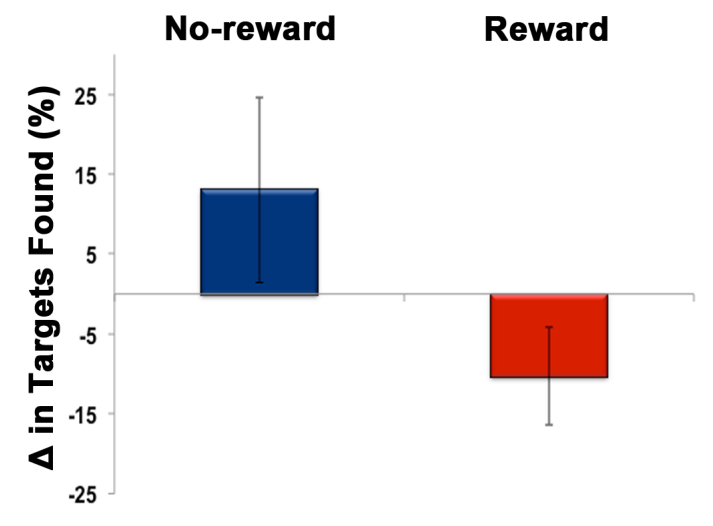

Figure 3 


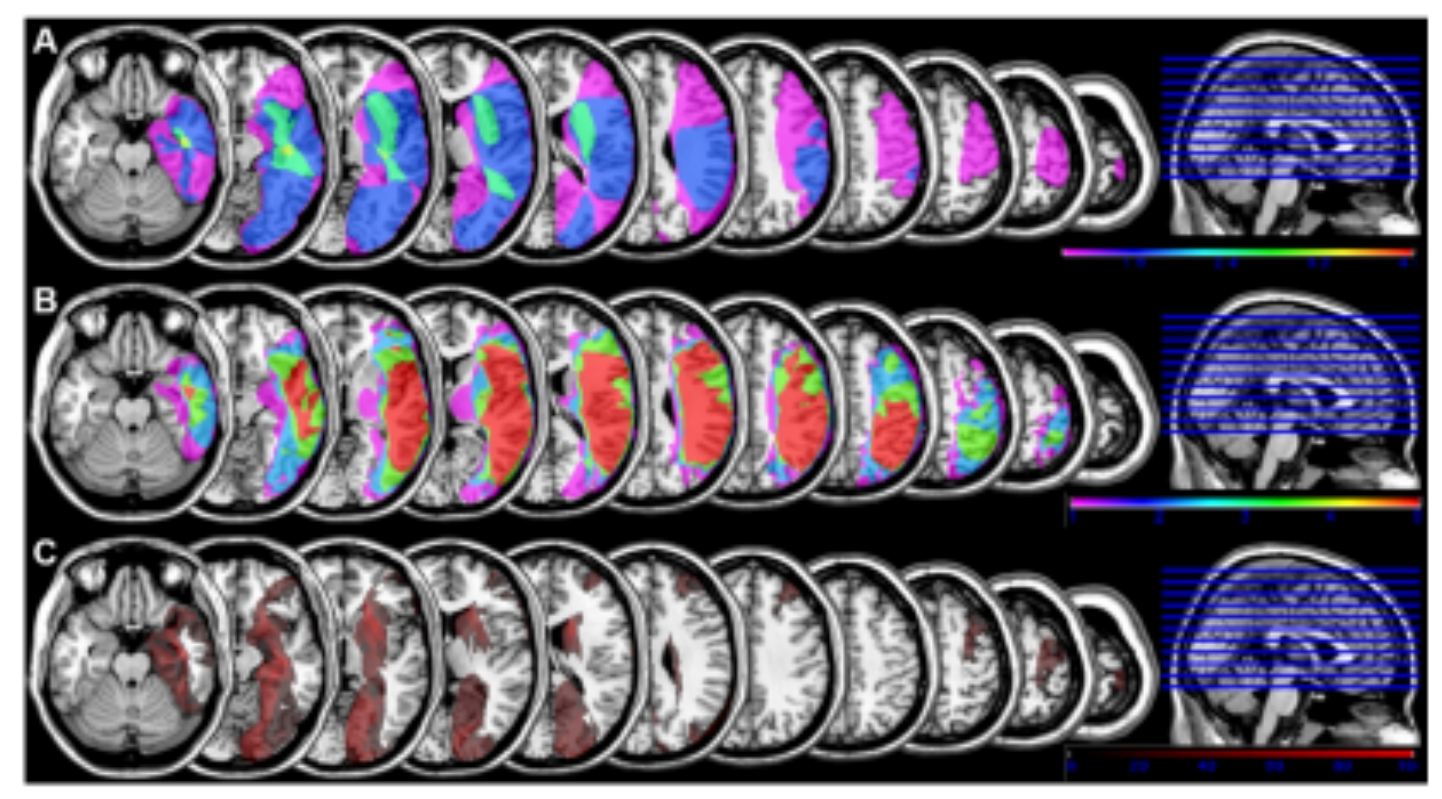

Figure 4 
A

B
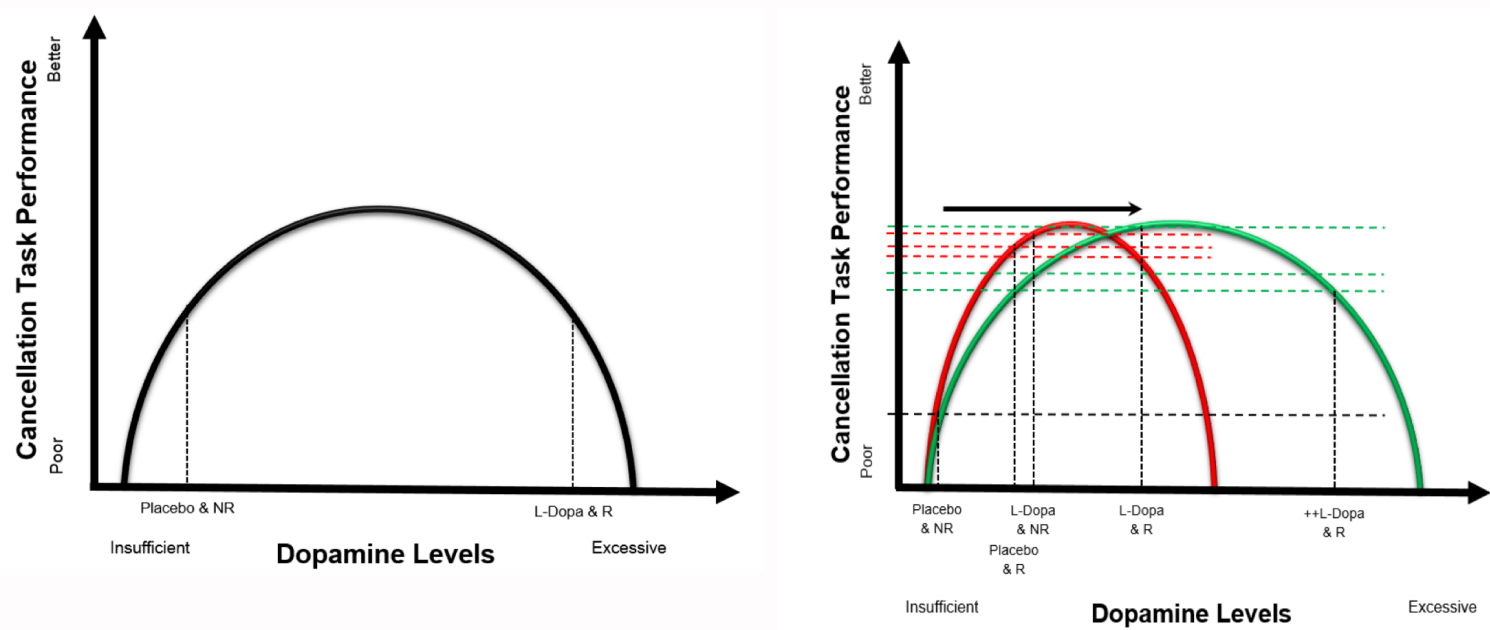

Figure 5 